\title{
采用柔性分子对接技术模拟蛋白质在 疏水作用色谱上的保留行为
}

\author{
周 鹏 ${ }^{\circledR}$ 田菲菲 ${ }^{\circledR}$ 李志良 ${ }^{\circledR} *$
}

(1) 重庆大学化学化工学院, 重庆 400044; (2) 化学生物传感与计量学国家重点实验室, 长沙 410082)

\begin{abstract}
摘要 将蛋白质与疏水作用色谱(HIC)固定相相互作用分为直接非键/构象作用和蛋白质表面 疏水效应两个热力学过程, 从而定量给出了处于浓盐析盐水溶液中 HIC 保留时间与配基/蛋白质 结合自由能之间的二元线性关系. 通过 ICM 柔性分子对接策略及遗传算法 (GA) 对 27 个已知晶体 结构的蛋白质与疏水配基的可能结合方式进行模拟和分析, 所得结果与实验观测情况吻合良 好. 研究表明, 蛋白质局部疏水效应以及配基与蛋白质的非键/构象作用皆对 HIC 色谱保留行为 影响显著, 且作用区域多集中于蛋白质表面突出部位.
\end{abstract}

\section{关键词 疏水作用色谱 柔性分子对接 遗传算法 蛋白质 盐溶因子}

疏水作用色谱(HIC) ${ }^{[1]}$ 是一种借助于偶联在固定 载体上的疏水基团与蛋白质(或核酸)表面发生疏水作 用而对样品组分进行分离的技术. HIC使用的流动相 通常是具有较高浓度的盐析盐水溶液, 在分离过程 中能够保持蛋白质分子的生物活性, 因此被广泛应 用于受体、血清蛋白、核蛋白、疫苗等生物分子的分 离纯化 ${ }^{[2]}$. 通常认为蛋白质在HIC中分离主要取决于 疏水, 而疏水作用被视为一种间接熵效应, 很难通过 一个确切的势能函数被规范化地表达出来; 另外, 柱 温、 $\mathrm{pH}$ 值、配基类型和密度、盐的种类及浓度等诸 多因素皆可影响色谱保留行为, 因此目前对于HIC分 离机制尚无一个统一认识. 为此人们提出了许多理 论来解释HIC保留机理, 如Arakawa的优先水化模型 [3]、Gelsema的熵增原理 [4]、Jennissen的多价吸附模型 [5]、Horváth的疏溶剂理论 ${ }^{[6]}$ 、Geng 的SDT-R ${ }^{[7]}$ 等. 近 年来部分学者将HIC研究兴趣转向了定量结构-色谱 保留关系(QSRR)途径: 即采用蛋白质分子的一级或 三级结构特征与其HIC保留时间建立统计学模型 ${ }^{[8,9]}$.
然而这些方法皆未考虑蛋白质在HIC分离过程中与 固定相配基相互作用及结合区域疏水性的影响, 仅 单方面从被分离对象的结构特征来研究保留行为的 差异, 这必将造成大量有用信息损失, 从而导致结果 的直观性和可解释性变差.

本文将浓盐溶液的影响及溶质水合因素引入到 通常药物与受体作用模型当中, 建立了 HIC 保留时 间与热力学效应之间的线性关系. 采用分子对接技 术模拟蛋白质表面可能的疏水结合区域, 并以遗传 算法最终优化及建立非键/构象作用和去溶剂化效应 与色谱保留时间的二元线性模型. 由 27 个已知晶体 结构蛋白质分析结果表明, 基于分子对接的遗传优 化方法能够较为准确地定义蛋白质表面结合区域, 并以此建立 HIC 保留时间与热力学参数之间的内在 关系, 所得线性模型回归系数正确反映了配基与蛋 白质结合过程的自由能变化方向. 同时还发现通常 蛋白质与配基发生结合区域的局部疏水特征和非键 作用对保留行为影响显著, 且往往处于蛋白质表面 
突出部位, 该结论与文献[10]报道一致.

\section{1 原理及方法}

\section{1 理论分析}

众多研究表明，蛋白质在HIC分离过程中与固定 相上配基的相互作用主要由疏水效应决定，其它非 键因素如静电作用、van der Waals势场、氢键等虽在 一定程度上影响着该过程的进行 ${ }^{[11,12]}$, 但它们的贡 献大小至今尚未明了. 由Horváth等 ${ }^{[6]}$ 的疏溶剂理论 可知, 处于极性流动相中的蛋白质表面疏水区域受 到溶剂排斥而被动与疏水配基发生结合，这个过程 起主导作用的是蛋白质和配基的共同疏水性. 当蛋 白质与配基发生进一步接触时，由于距离的拉近以 及阻隔溶剂排出，二者之间将同时伴随其它非键效 应发生. 进一步考虑到蛋白质通过HIC色谱柱是一个 吸附和解吸的动态平衡过程, 由于蛋白质分子远远 大于固定相配基，表面存在诸多可以与配基发生可 逆结合的区域. 由此我们认为蛋白质表面所有结合 区域对整个分离过程平均自由能变化的贡献如下:

$$
\begin{aligned}
\Delta \bar{G}_{\mathrm{bind}} & =\Delta \bar{G}_{\mathrm{int}}+\Delta \bar{G}_{\mathrm{sol} \downarrow} \\
& =\frac{1}{n} \sum_{i=1}^{n} \Delta G_{\mathrm{int}}^{i}+\frac{1}{n} \sum_{i=1}^{n}\left(\Delta G_{\mathrm{P}, \mathrm{sol} \downarrow}^{i}+\Delta G_{\mathrm{L}, \mathrm{sol} \downarrow}^{i}\right),
\end{aligned}
$$

其中 $n$ 为蛋白质表面能够与配基发生作用的疏水区 域数目; $\Delta G_{\text {int }}^{i}$ 是第 $i$ 个疏水区域与配基直接作用的 自由能变化, 它由非键作用自由能和构象改变自由 能两个方面组成 (下文统称非键/构象自由能); $\left(\Delta G_{\mathrm{P}, \mathrm{sol} \downarrow}^{i}+\Delta G_{\mathrm{L}, \mathrm{sol} \downarrow}^{i}\right)$ 为第 $i$ 个疏水区域与配基结合时 的整体去溶剂化自由能变化大小(下标 $\mathrm{P}$ 和 $\mathrm{L}$ 分别代 表蛋白质和配基，】表示去溶剂化过程，下文个表示水 合过程).

按照Eisenberg ${ }^{[13]}$ 和Ooi ${ }^{[14]}$ 等的纯水溶液中溶剂化 参数理论，可以定义蛋白质表面结合区域 $i$ 的水合自由 能变化为该区域所有 $r_{i}$ 个氨基酸残基疏水性 $(\sigma)$ 与其暴 露在外的溶剂可及面积 $(A)$ 的乘积之和. 当溶剂为浓盐 析盐水溶液时, Arakawa等 ${ }^{[3]}$ 的优先水化模型指出, 此 时蛋白质表面会倾向于形成一层水化膜，而阻止盐同 蛋白质发生接触，盐的存在只是降低了水分子结合蛋 白质疏水表面的能力, 其自身并不直接与蛋白质发生 作用. 即蛋白质与配基结合时其疏水表面失去的溶剂 仅为水分子, 而不涉及到盐离子. 鉴于此, 我们认为
在HIC的浓盐析盐水溶液环境中蛋白质结合区域的去 溶剂化自由能变化需要在纯水溶液情况下的计算公式 前面引入一个盐溶因子 $\lambda$, 以表明盐的存在对水分子 脱离蛋白质疏水表面的贡献 $(\lambda$ 取值与盐的种类以及离 子强度有关), 即 $\Delta G_{\mathrm{P}, \mathrm{sol} \downarrow}^{i}=-\Delta G_{\mathrm{P}, \mathrm{sol} \uparrow}^{i}=-\lambda \sum_{j}^{r_{i}} \sigma_{j}^{i} A_{j}^{i}$ ( $\Delta G_{\mathrm{P}, \mathrm{sol} \uparrow}^{i}$ 代表水合自由能，负号表示水合过程是去溶 剂化的逆向变化), 将其代入式(1)可得:

$$
\Delta \bar{G}_{\mathrm{bind}}=\frac{1}{n} \sum_{i=1}^{n} \Delta G_{\mathrm{int}}^{i}-\frac{\lambda}{n} \sum_{i=1}^{n} \sum_{j=1}^{r_{i}} \sigma_{j}^{i} A_{j}^{i}+\frac{1}{n} \sum_{i=1}^{n} \Delta G_{\mathrm{L}, \mathrm{sol} \downarrow}^{i}
$$

在一次多组分蛋白质样品分离过程中由于 HIC 固定相配基是不变的，因此可以认为引起不同种类 蛋白质发生分离的主要因素为式(2)中前两个部分, 即蛋白质与配基非键/构象自由能及蛋白质结合区域 去溶剂化自由能，而第 3 部分配基去溶剂化自由能变 化可视为常数. 进一步假设保留时间 $(R T)$ 与蛋白质和 固定相配基作用的平均自由能变化成线性关系，即 $R T=a_{0}+a_{1} \times \Delta \bar{G}_{\text {bind }}$, 将式(2)代入其中, 并合并常数 项后即得下式:

$$
\begin{aligned}
R T & =a_{0}^{\prime}+a_{1} \Delta \bar{G}_{\mathrm{int}}-a_{1}^{\prime} \Delta \bar{G}_{\mathrm{P}, \mathrm{sol} \uparrow} \\
& =a_{0}^{\prime}+a_{1}\left(\frac{1}{n} \sum_{i=1}^{n} \Delta G_{\mathrm{int}}^{i}\right)-a_{1}^{\prime}\left(\frac{1}{n} \sum_{i=1}^{n} \sum_{j=1}^{r_{i}} \sigma_{j}^{i} A_{j}^{i}\right),
\end{aligned}
$$

其中 $a_{0}^{\prime}=a_{0}+a_{1}\left(\frac{1}{n} \sum_{i=1}^{n} \Delta G_{\mathrm{L}, \mathrm{sol} \downarrow}^{i}\right), a_{1}^{\prime}=a_{1} \lambda ; \Delta \bar{G}_{\text {int }}$ 和 $\Delta \bar{G}_{\mathrm{P}, \mathrm{sol} \uparrow}$ 分别为配基与蛋白质的平均非键/构象自由 能及蛋白质表面结合区域的平均水合自由能(注意: 由于盐溶因子 $\lambda$ 已被归入系数项 $a_{1}^{\prime}$ 中, 故这里 $\Delta \bar{G}_{\mathrm{P}, \mathrm{sol} \uparrow}$ 为纯水溶液中的平均水合自由能).

\section{2 分子对接采样}

通常药物与生物大分子对接过程是在已经定义 好的活性位点进行, 如蛋白质口袋、裂痕、孔洞等, 与 之不同的是 HIC中配基与待分离蛋白质表面的许多 区域都可能发生作用, 并且这些区域并不一定是蛋 白质的凹陷位置, 事实证明由于位阻效应和表面暴 露等原因使得配基更倾向于与蛋白质表面突出部位 发生接触 ${ }^{[14]}$. 鉴于此, 在进行分子对接模拟时需要 对目标蛋白的整个表面空间进行分析. 为了提高计 算效率并保证结果精度，本文使用 Totrov等 [15]提出 
的 ICM 方法实现分子对接采样. ICM 是一种基于 Monte Carlo结合局部优化技术的全柔性分子对接方 法, 它通过计算配基与受体之间的静电、立体、氢键 作用能以及二者因单键旋转引起的构象自由能变化 来评价对接结果. 在ICM模型中允许配基及其附近受 体侧链和柔性主链的单键自由旋转, 但键长和键角 固定不变, 这样就在柔性机制和计算效率间达成了 较好的平衡. 同时为了避免随着自由度增加导致 Monte Carlo搜索空间过大, ICM对接过程中采用分子 内坐标代替传统笛卡尔坐标, 从而极大限度地提高 了计算速度.

\section{3 氨基酸疏水参数}

自从Tanford等 [16]为疏水作用的存在提供了实验 数据以来, 至今已有数百种与氨基酸残基水溶性相 关的参数被相继提出. 但是这些数据都是通过间接 方法测得(如色谱保留值、分配系数、疏水矩等), 从 单位和形式上来看都不能直接代入本文溶剂化参数 模型中, 为此在这里我们专门提出了一套与该模型 相匹配的氨基酸疏水参数.

Ooi等 [14] 认为, 一个处于溶解状态的溶质分子只 能与其周围一层水分子发生作用，这种作用强弱与 分子自身的疏水性以及与水分子的接触面积有关, 故定义分子水合自由能为 $\Delta G=\sigma A$. 其中 $\sigma$ 为溶质的 疏水参数; $A$ 为溶剂可及面积，是以水分子(通常可视 为van der Waals半径 $1.4 \AA$ 的球体)为探针在分子表面 滚动其球心形成的表面面积. 很显然, 如果已知 $\Delta G$ 和 $A$ 就可以通过该式计算分子的疏水参数 $\sigma$. 由于物 质从气相进入液相发生溶解相当于一个被溶剂化的 过程，该过程体系自由能变化包括水合自由能及分 子自身构象变化能. 通常对于小分子而言, 溶解过程 构象变化引起的自由能改变可以忽略，故可将分子 的溶解自由能视为其水合自由能大小. 鉴于此, 本文 氨基酸的水合自由能取文献[12]定义的相对溶解自由 能 $\Delta G=1.36 \times[\lg P(\mathrm{X})-\lg P(\mathrm{Gly})]$; 另外, 对于氨基酸 溶剂可及面积, 当前使用较为广泛的是由Rose等 ${ }^{[17]}$ 测 得的残基 X处在三肽Gly-X-Gly中的取值，虽然这个 数值并不是孤立氨基酸分子的溶剂可及面积, 但是 由于两边紧邻甘氨酸残基没有侧链, 因此其值较为 接近分子真实状态，故为本文所用. 20 种天然氨基酸 的相对溶解自由能 $\Delta G$, 溶剂可及面积 $A$ 以及由此计 算得到的疏水参数 $\sigma$ 参见表 1 .
表 120 个标准天然氨基酸的相对溶解自由能 $\Delta G$, 溶剂可 及面积 $A$ 和疏水参数 $\sigma^{[17]}$

\begin{tabular}{|c|c|c|c|c|}
\hline 编号 & 氨基酸 & $\Delta G / \mathrm{kcal} \cdot \mathrm{mol}^{-1 \mathrm{a})}$ & $A / \AA$ & $\begin{array}{c}10^{2} \sigma / \\
\mathrm{kcal} \cdot \mathrm{mol}^{-1} \cdot \AA^{-1 \mathrm{a})}\end{array}$ \\
\hline 1 & Ala, A & 0.42 & 118.1 & 0.36 \\
\hline 2 & Arg, R & -1.37 & 256.0 & -0.54 \\
\hline 3 & Asn, N & -0.82 & 165.5 & -0.50 \\
\hline 4 & Asp, D & -1.05 & 158.7 & -0.66 \\
\hline 5 & Cys, C & 1.34 & 146.1 & 0.92 \\
\hline 6 & Gln, Q & -0.30 & 193.2 & -0.16 \\
\hline 7 & Glu, E & -0.87 & 186.2 & -0.47 \\
\hline 8 & Gly, G & 0.00 & 88.1 & 0.00 \\
\hline 9 & His, $\mathrm{H}$ & 0.18 & 202.5 & 0.09 \\
\hline 10 & Ile, I & 2.46 & 181.0 & 1.36 \\
\hline 11 & Leu, L & 2.32 & 193.1 & 1.20 \\
\hline 12 & Lys, K & -1.35 & 225.8 & -0.60 \\
\hline 13 & Met, M & 1.68 & 203.4 & 0.83 \\
\hline 14 & Phe, F & 2.44 & 222.8 & 1.10 \\
\hline 15 & Pro, $\mathrm{P}$ & 0.98 & 146.8 & 0.67 \\
\hline 16 & Ser, S & -0.05 & 129.8 & -0.04 \\
\hline 17 & Thr, T & 0.35 & 152.5 & 0.23 \\
\hline 18 & Trp, W & 3.07 & 266.3 & 1.15 \\
\hline 19 & Tyr, Y & 1.31 & 236.8 & 0.55 \\
\hline 20 & Val, V & 1.66 & 164.5 & 1.01 \\
\hline
\end{tabular}

a) $1 \mathrm{cal}=4.1868 \mathrm{~J}$

\section{4 实验部分}

27 个已知晶体结构蛋白质的PDB代码及经由梯 度延迟效应和柱死体积校正的 HIC 保留时间 $R T_{\mathrm{Obsd}}$ 参 见表 2. 实验方法 ${ }^{[18]}$ : 采用高效液相色谱仪带梯度洗 脱洜、自动进样阀和二极管阵列光度检测器(测定波 长 $280 \mathrm{~nm}$ ), 固定相为苯基-交联琼脂糖凝胶. 在室温 及 $\mathrm{pH}=7.0$ 条件下进行实验，测定时取 $100 \mu \mathrm{g}$ 的样品 注射入 $100 \mathrm{~mm} \times 5 \mathrm{~mm} \mathrm{HIC}$ 色谱柱并执行 $90 \mathrm{~min}$ 梯度 洗提, 该过程中流动相流速为 $0.5 \mathrm{~mL} / \mathrm{min}$, 溶液的硫 酸铵盐浓度由 $1.4 \mathrm{~mol} / \mathrm{L}$ 线性递减为 $0 \mathrm{~mol} / \mathrm{L}$, 之后保 持恒定条件 $10 \mathrm{~min}$ 以去除柱内结合稳固的样品.

\section{5 计算过程}

样本集划分：在 27 个样本中随机抽取 4 个蛋白 质(表 2 中以黑体表示)不参与先期计算, 将被用于检 测所得线性模型的性能.

配基准备：从苯基-交联琼脂糖凝胶固定相上截 取的配基包含苯基及醚酯键连接部分，以氢原子封 端基. 使用理论化学软件 HyperChem 7.5 构建并初步 优化(MM+力场)得到的配基分子立体结构，进一步 采用量子化学软件包 Gaussian $03 \mathrm{~W}$ 在密度泛函水平 (B3LYP/6-31G**)进行 Berny 几何全优化至收敛, 所 得稳定结构经振动分析无虚频存在. 使用自然布居 
表 227 个样本蛋白质的相关数据 ${ }^{[18]}$

\begin{tabular}{|c|c|c|c|c|c|c|}
\hline 编号 & 蛋白质 & PDB & $\Delta \bar{G}_{\mathrm{int}} / \mathrm{kcal} \cdot \mathrm{mol}^{-1 \mathrm{a})}$ & 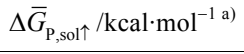 & $R T_{\text {观测值 }} / \mathrm{min}$ & $R T_{\text {计算值 }} / \mathrm{min}$ \\
\hline 1 & 核糖核酸酶 B & $1 \mathrm{RBJ}$ & -19.79 & -7.32 & 3.57 & 8.02 \\
\hline 2 & 核糖核酸酶 $\mathrm{A}$ & $1 \mathrm{AQP}$ & -18.97 & -4.66 & 5.52 & 14.07 \\
\hline 3 & 碳酸酐酶 & $1 \mathrm{HCB}$ & -16.34 & -0.41 & 15.86 & 20.79 \\
\hline $4^{\Delta}$ & 红氧还蛋白 & 1FHM & -24.58 & -1.51 & 24.18 & 35.99 \\
\hline 5 & 菠萝蛋白酶抑制剂 & 1BI6 & -26.13 & -1.12 & 40.03 & 40.62 \\
\hline 6 & 门冬酰胺酶 & $3 \mathrm{ECA}$ & -21.94 & 3.61 & 44.72 & 45.27 \\
\hline 7 & 卵清溶菌酶 & $1 \mathrm{AKI}$ & -22.77 & 4.48 & 59.11 & 49.71 \\
\hline 8 & 火鸡卵清溶菌酶 & $135 \mathrm{~L}$ & -25.56 & 4.16 & 66.42 & 55.01 \\
\hline 9 & 胰蛋白酶原 & 1TGB & -28.10 & 5.39 & 72.13 & 64.35 \\
\hline $10^{\Delta}$ & 扁豆凝集素 & 2LAL & -31.85 & 7.97 & 69.85 & 80.41 \\
\hline 11 & $\alpha$-糜蛋白酶 & 4CHA & -30.81 & 6.82 & 91.09 & 74.67 \\
\hline 12 & 胰蛋白酶抑制剂 & $1 \mathrm{AVU}$ & -28.12 & 6.16 & 71.05 & 66.68 \\
\hline 13 & $\gamma$-糜蛋白酶 & $2 \mathrm{GCH}$ & -29.56 & 6.91 & 84.47 & 72.14 \\
\hline 14 & 丙酮酸激酶 & $1 \mathrm{AQF}$ & -32.41 & 7.79 & 91.58 & 81.13 \\
\hline $15^{\Delta}$ & $\alpha$-糜蛋白酶原 A & $1 \mathrm{EX} 3$ & -23.69 & 6.29 & 78.12 & 57.14 \\
\hline 16 & 卵清蛋白 & 1OVA & -28.80 & 7.21 & 56.92 & 71.32 \\
\hline 17 & 谷草转氨酶 & 1AJR & -29.12 & 8.80 & 74.31 & 76.76 \\
\hline 18 & 前白蛋白 & 1RLB & -23.25 & 6.83 & 52.61 & 57.76 \\
\hline 19 & 小白蛋白 & $1 \mathrm{RTP}$ & -27.21 & 8.07 & 62.73 & 70.31 \\
\hline 20 & 枯草杆菌蛋白酶 & $1 \mathrm{CSE}$ & -32.53 & 9.62 & 92.72 & 86.83 \\
\hline $21^{\Delta}$ & 蜂毒磷脂酶 A2 & 1POC & -30.82 & 8.54 & 92.57 & 79.80 \\
\hline 22 & $\beta$-乳球蛋白 A & $1 \mathrm{~B} 0 \mathrm{O}$ & -33.65 & 10.39 & 90.91 & 91.63 \\
\hline 23 & 过氧化氢酶 & 4BLC & -29.20 & 13.21 & 95.00 & 90.02 \\
\hline 24 & 脂肪酶 & 3TGL & -33.53 & 12.10 & 91.29 & 96.43 \\
\hline 25 & 枯草溶菌蛋白酶 & $1 \mathrm{AF} 4$ & -36.25 & 11.75 & 92.16 & 101.49 \\
\hline 26 & －乳白蛋白 & $1 \mathrm{~F} 6 \mathrm{~S}$ & -35.13 & 11.98 & 88.66 & 99.66 \\
\hline 27 & 胰岛素 & 4INS & -36.83 & 12.18 & 95.89 & 104.07 \\
\hline
\end{tabular}

a) $1 \mathrm{cal}=4.1868 \mathrm{~J}$

分析法赋予原子偏电荷. 需要说明的是，由于 ICM 采 用柔性对接策略，因此这里优化得到的配基分子立 体结构仅为初始构象, 其在对接过程中将发生改变.

对接采样: 所有样本蛋白质晶体结构都在药物/ 生物系统分析软件包Molsoft ICM 3.3 中重新构建. 过程如下：对所输入的蛋白质去除结晶水、添加/优化 氢原子并采用Maiorov法 [19]进行结构诊断，在此基础 上执行柔性区域结构调整和侧链优化配置处理, 所 得结果以 $\mathrm{icm}$ 目标文件格式保存(*.ob). ICM分子对接 由 docking模块实现，采样区域定义为整个蛋白质表 面空间, 辅以格点势能法来加快对接自由能计算速 度(格点间距 $0.5 \AA$ ). 执行完毕后对每个蛋白质样本 取得分最高的前 30 个配基/蛋白质复合构象作为可能 的结合方式.

遗传优化：在分子对接给出的配基/蛋白质多重 复合构象的基础上进一步使用遗传算法 $(\mathrm{GA})$ 精确篎 选蛋白质表面与配基的作用区域. 对余下 23 个蛋白 质样本进行处理，具体实现如下：每个蛋白质都由一 个长度为 30 的二进制(短)串表示, (短)串中各个位点
分别代表由ICM分子对接给出的 30 种可能的配基/蛋 白质结合方式，当其值取 1 表示选中该复合构象, 0 则 表示末选中. 将 23 个样本的(短)串依次连接成一个 (长) 串, 其长度为 $23 \times 30=690$. 这个(长) 串被视为 $\mathrm{GA}$ 中一个个体的染色体，其每个位点则为该个体的基 因. 在执行GA迭代过程时，每个个体中被选中的基 因共同构成 23 个蛋白质与配基的可能结合方式, 即 每个蛋白质样本都有数种配基/蛋白质复合构象被挑 选. 计算出 23 个样本蛋白各自被选中复合构象的平 均非键/构象自由能 $\Delta \bar{G}_{\text {int }}$ 和表面结合区域平均水合 自由能 $\Delta \bar{G}_{\mathrm{P}, \mathrm{sol} \uparrow}$ (每种复合构象的非键/构象自由能已 由分子对接给出; 水合自由能采用溶剂化参数模型 计算，定义复合物中配基周围 $5 \AA$ 迪内的氨基酸残基 为有效范围, 它们各自处于折叠蛋白质中的溶剂可 及面积预先提交由ASC服务器 [20]计算得到), 将每个 蛋白质计算所得的平均非键/构象自由能和平均水合 自由能代入式(3), 由这 23 个样本蛋白进行二元线性 拟合得到回归系数 $a_{0}^{\prime}, a_{1}, a_{1}^{\prime}$ 及均方根误差(RMS), 并 以 $\mathrm{RMS}$ 作为 $\mathrm{GA}$ 个体的适应度评价函数. 这样经过 
GA反复执行选择、交叉、变异算子, 最终演化产生 一个优秀种群，取该种群最优个体进行分析.

\section{2 结果与分析}

经过 GA 计算所得最优个体参数: $a_{0}^{\prime}=-14.619, a_{1}=$ $-2.241, a_{1}^{\prime}=-2.967$, 将其代入式(3)确定二元线性回归 关系: $R T=-14.619-2.241 \times \Delta \bar{G}_{\mathrm{int}}+2.967 \times \Delta \bar{G}_{\mathrm{P}, \mathrm{sol} \uparrow}$. 该 方程对 23 个蛋白质样本的拟合复相关系数 $R$ 及均方 根误差 RMS 分别为 0.955 和 8.355 , 从图 1 可见模型 计算值与实验值吻合较好，没有异常情况. 对回归方 程的系数项分析表明, 平均非键/构象自由能 $\Delta \bar{G}_{\mathrm{int}}$ 及 平均水合自由能 $\Delta \bar{G}_{\mathrm{P}, \mathrm{sol} \uparrow}$ 与色谱保留时间 $R T$ 分别呈 负相关和正相关关系. 这是由于 $\Delta \bar{G}_{\text {int }}$ 表示配基与蛋 白质直接作用强度 $\left(\Delta \bar{G}_{\text {int }}\right.$ 取值与作用强度呈负向变 化), 而作用强度又直接影响保留时间(作用强度与保 留时间呈正向变化); 另外 $\Delta \bar{G}_{\mathrm{P}, \mathrm{sol} \uparrow}$ 代表蛋白质结合区 域的疏水性 $\left(\Delta \bar{G}_{\mathrm{P}, \mathrm{sol} \uparrow}\right.$ 取值与疏水性呈正向变化), 显 然具有强区域疏水性的蛋白质因易于脱除表面水合 层而有利于与配基结合，从而增强其在色谱柱上的 保留能力(即 $R T$ 增大). 由上述分析可知，本文模型系 数项与实际情况相符, 其较好解释了 HIC 色谱柱上 配基与蛋白质结合时的两类自由能变化对保留时间 的影响. 表 2 列出了经 $\mathrm{GA}$ 优化得到的平均非键/构象 自由能 $\Delta \bar{G}_{\mathrm{int}}$ 及平均水合自由能 $\Delta \bar{G}_{\mathrm{P}, \mathrm{sol} \uparrow}$ 取值, 观察发 现所有样本的 $\Delta \bar{G}_{\text {int }}$ 皆小于零, 而绝大多数样本的 $\Delta \bar{G}_{\mathrm{P}, \mathrm{sol} \uparrow}$ 则大于零. 故可认为通常 HIC 色谱柱中固定 相配基与蛋白质发生结合的位置在满足疏水性的前 提下更倾向于选择有利于二者发生直接作用的区域. 图 2 和图 3 分别为 $\Delta \bar{G}_{\mathrm{int}}$ 及 $\Delta \bar{G}_{\mathrm{P}, \mathrm{sol} \uparrow} \uparrow$ 单独与 $R T$ 的相关 情况, 可见 $\Delta \bar{G}_{\mathrm{int}}$ 和 $\Delta \bar{G}_{\mathrm{P}, \mathrm{sol} \uparrow}$ 与 $R T$ 具有良好的线性关 系 $(R$ 分别为 -0.886 及 0.929$)$, 且 $\Delta \bar{G}_{\mathrm{P}, \text { sol } \uparrow}$ 相关性优于 $\Delta \bar{G}_{\text {int }}$. 由此我们认为, 虽然疏水作用在 HIC 中起主 导地位，但是配基与蛋白质的非键/构象作用也扮演 着较为重要的角色. 进一步分析盐溶因子 $\lambda$ 的影响情 况，本文定义的盐溶因子 $\lambda$ 表示由于盐的加入促使水 分子脱离蛋白质疏水表面的趋势大小，由式(3)可知 $\lambda=a_{1}^{\prime} / a_{1}$. 这里色谱系统的 $a_{1}^{\prime}$ 和 $a_{1}$ 已由上文 $\mathrm{GA}$ 优化 得到, 故可计算出 $\lambda=1.324$. 将 $\lambda$ 放入经其校正的溶
剂化参数模型 $\Delta G_{\mathrm{P}, \mathrm{sol} \uparrow}=\lambda \sum_{i} \sigma_{i} A_{i}$ 进行讨论：对于疏 水区域的 $\sum_{i} \sigma_{i} A_{i}$ 取值为正，而本文利用线性拟合得 到的模型系数计算出 $\lambda=1.324>1$, 由此表明由于盐 的存在增大了蛋白质表面结合区域的疏水性，并且 这种效应可以定量地由 $\lambda$ 给出.

以过氧化氢酶为例(PDB 10 为 4BLC), 该蛋白质 由 4 个亚基组成, 其实验及计算 $R T$ 值皆大于 90 , 具 有较强的色谱保留能力. 图 4 给出了一种较为典型的

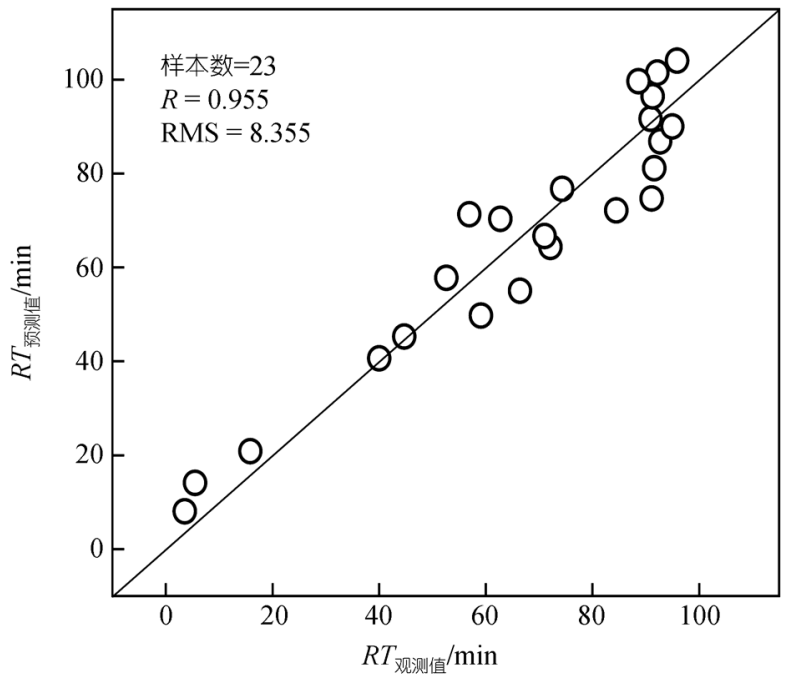

图 123 个样本蛋白质色谱保留时间 $R T$ 的计算值与实验 值相对情况

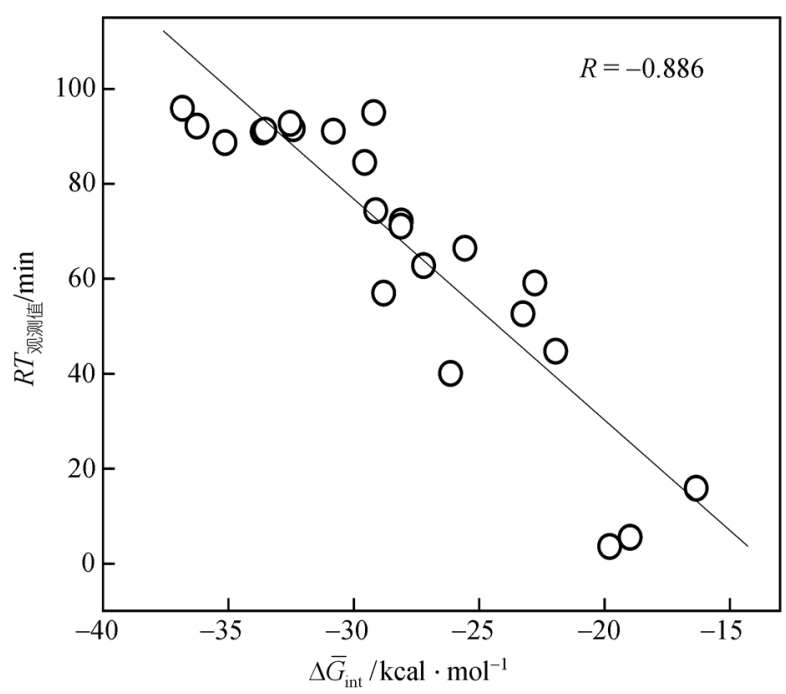

图 2 平均非键/构象自由能 $\Delta \bar{G}_{\mathrm{int}}$ 与色谱保留时间 $R T$ 的相 关性 


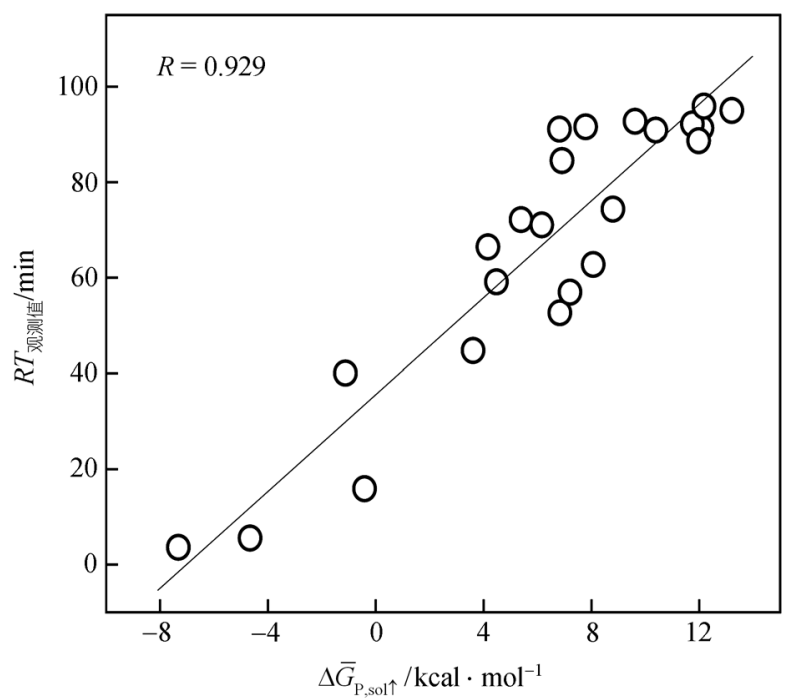

图 3 平均水合自由能 $\Delta \bar{G}_{\mathrm{P}, \mathrm{sol} \uparrow}$ 与色谱保留时间 $R T$ 的 相关性

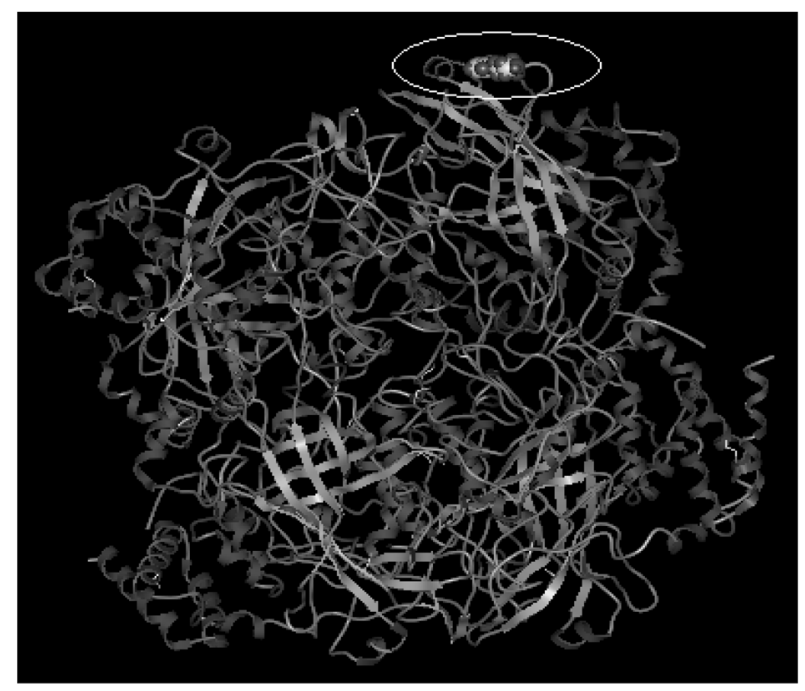

图 4 较为典型的配基/蛋白质结合方式

配基/蛋白质结合方式，可以看到配基分子嵌于蛋白 质表面一个突出区域，并分别被 $\alpha$ 螺旋、 $\beta$ 折叠和无 规卷曲所环绕. 事实上我们观察发现经GA选取的结 合区域多数都处于(或接近)蛋白质表面突起部分(约 $80 \%$ ), 很少出现在裂缝和山陷口袋中, 这与药物和受 体作用方式截然相反. 可以认为出现这种情况是由 于蛋白质在 HIC 中呈快速吸附/解吸动态平衡状态, 其表面突出部位有更多机会与固定相配基发生接触, 这与Mahn等 ${ }^{[10]}$ 研究核糖核酸酶 $\mathrm{A}$ 在HIC 上保留行为 所得结论一致. 图 5 展示了结合部位的详细情况,

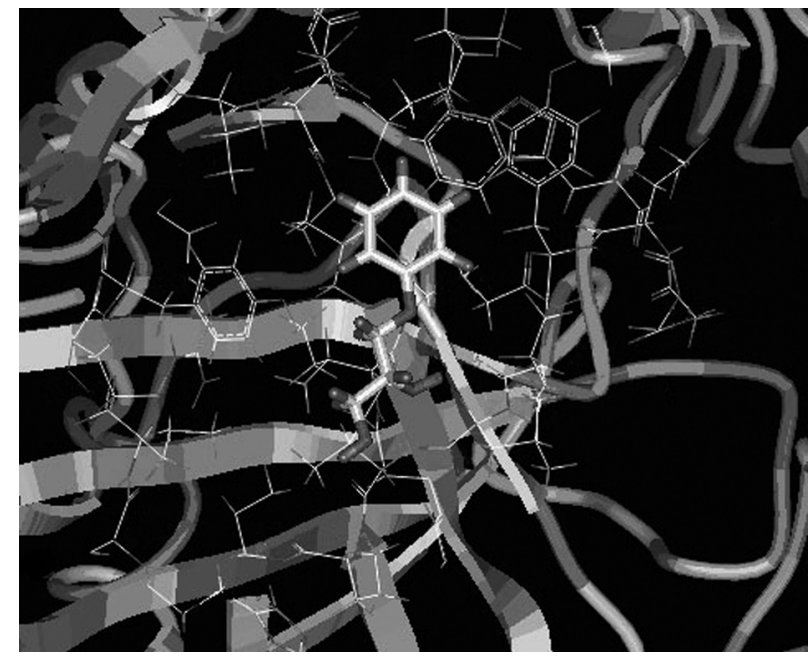

图 5 配基/蛋白质结合部位的详细情况

可以看到在配基周围环绕了较多疏水性氨基酸残基 (同时也存在部分两亲性和亲水性氨基酸), 如带有烃 基侧链的 $\mathrm{Ile}_{90}, \mathrm{Leu}_{221}, \mathrm{Val}_{229}, \mathrm{Ile}_{280}, \mathrm{Val}_{282}, \mathrm{Pro}_{308}$, $\mathrm{Leu}_{309}, \mathrm{Ile}_{310}, \mathrm{Pro}_{311}, \mathrm{Val}_{312}$ 以及带有芳环结构的 $\mathrm{Tyr}_{93}$, $\mathrm{Tyr}_{230}, \mathrm{Phe}_{291}, \mathrm{Trp}_{302}, \mathrm{Tyr}_{307}$ 等, 它们共同构成蛋白质 表面局部疏水区域. 另外，经深入分析发现，配基分 子的苯环可与周围残基 $\mathrm{Tyr}_{93}, \mathrm{Trp}_{302}, \mathrm{Tyr}_{307}$ 侧链芳环 产生微弱的 $\pi$ 键堆积效应, 而尾部一个羟基中的氢原 子则与 $\mathrm{Ile}_{310}$ 主链上的羰基氧原子发生氢键作用(键长 $0.27 \mathrm{~nm}$, 键角 $\left.134^{\circ} 26^{\prime}\right)$. 这些现象表明了非键效应在 HIC 分离过程中起到了重要的作用.

综上研究表明, HIC 中固定相配基与蛋白质作用 通常满足以下三点：(1) 对接非键/构象自由能小于 $-62.7 \mathrm{~kJ} / \mathrm{mol}$; (2) 蛋白质表面结合区域的水合自由能 大于 $-41.8 \mathrm{~kJ} / \mathrm{mol}$; (3) 结合区域不处于蛋白质的口袋 或裂缝当中. 按照该规律对余下 4 个测试蛋白质样本 进行分析，在 ICM 对接给出的 30 种可能的配基/蛋白 质复合构象中分别在每个蛋白质表面选取了 $11,17,14$, 21 个疏水结合区域，按照这些位置计算出样本蛋白平 均非键/构象自由能 $\Delta \bar{G}_{\text {int }}$ 及平均水合自由能 $\Delta \bar{G}_{\mathrm{P}, \mathrm{sol} \uparrow}$ 代入二元线性回归方程 $R T=-14.619-2.241 \times \Delta \bar{G}_{\mathrm{int}}+$ $2.967 \times \Delta \bar{G}_{\mathrm{P}, \mathrm{sol} \uparrow}$ 中预测得到的色谱保留时间 $R T$ 与实 验值吻合良好, 4 个样本的 RMS 仅为 14.614.

\section{3 结论}

当前人们通常从热力学和 QSRR 途径来阐述 $\mathrm{HIC}$ 保留行为, 本文将药物设计领域的柔性分子对接 
技术引入到 $\mathrm{HIC}$ 模拟当中，利用 GA 成功构建了配基 /蛋白质相互作用平均非键/构象自由能和蛋白质表面 结合区域平均水合自由能与色谱保留时间的二元线 性关系. 通过对 27 个已知晶体结构蛋白质在 HIC 中 保留行为的模拟结果表明: (1) 配基与蛋白质作用过 程不仅受疏水效应影响，非键/构象作用也扮演了较 为重要的角色；(2) 配基与蛋白质结合主要发生在蛋 白质表面突出部位；(3) 浓盐析盐溶液对蛋白质表面 疏水性的增强可以通过盐溶因子 $\lambda$ 定量给出.

\section{参考文 献}

1 Queiroz J A, Tomaz C T, Cabral J M S. Hydrophobic interaction chromatography of proteins. J Biotech, 2001, 87: 143-159[DOI]

2 Glick D. Methods of Biochemical Analysis. New York: John Wiley \& Sons, 1981. 89-108

3 Arakawa T. Thermodynamic analysis of the effect of concentrated salts on protein interaction with hydrophobic and polysaccharide columns. Arch Biochem Biophys, 1986, 248(1): 101-105[DOI]

4 Gelsema W J, Brandts P M, Deligny C L, Theeuwes A G M, Roozen A M P. Hydrophobic interaction chromatography of aliphatic alcohols and carboxylic acids on octyl-sepharose CL-4B: mechanism and thermody-namics. J Chromatogr, 1984, 295: 13-29[DOI]

5 Jennissen H P. Multivalent interaction chromatography as exemplified by the adsorption and desorption of skeletal muscle enzymes on hydrophobic alkyl-agaroses. J Chromatogr, 1978, 159: $71-83$

6 Melander W, Horváth C. Salt effects on hydrophobic interactions in precipitation and chromatography of proteins: an interpretation of the lyotropic series. Arch Biochem Biophys, 1977, 183(1): 200-215

7 Geng X D, Guo L A, Chang J H. Study of the retention mechanism of protein in hydrophobic interaction chromatograohy. J Chromatogr, 1990, 507: 1-23[DOI]
8 Mazza C B, Sukumar N, Breneman C M, Cramer S M. Prediction of protein retention in ion-exchange systems using molecular descriptors obtained from crystal structure. Anal Chem, 2001, 73: 5457-5461[DOI]

9 Salgado J C, Rapaport I, Asenjo J A. Prediction of retention times of proteins in hydrophobic interaction chromatography using only their amino acid composition. J Chromatogr A, 2005, 1098: 44-54[DOI]

10 Mahn A, Lienqueo M E, Asenjo J A. Effect of surface hydrophobicity distribution on retention of ribonucleases in hydrophobic interaction chromatography. J Chromatogr A, 2004, 1043: 47-55[DOI]

11 Hjertén S. Some general aspects of hydrophobic interaction chromatography. J Chromatogr, 1973, 87: 325-331

12 Van Oss C J, Good R J, Chaudhury M K. Nature of the antigen-antibody interaction. Primary and secondary bonds: optimal conditions for association and dissociation. J Chromatogr, 1986, 376: 111-119[DOI]

13 Eisenberg D, McLachlan A D. Solvation energy in protein folding and binding. Nature, 1986, 319: 199-203[DOI]

14 Ooi T, Oobatake M, Nmmethy G, Harold A, Scheraga H A. Accessible surface areas as a measure of the thermodynamic parameters of hydration of peptides. Proc Natl Acad Sci USA, 1987, 84: 3086-3090[DOI]

15 Abagyan R A, Totrov M M, Kuznetsov D A. ICM: a new method for structure modeling and design: applications to docking and structure prediction from the distorted native conformation. J Comp Chem, 1994, 15: 488-506[DOI]

16 Tanford C. The hydrophobic effect and the organization of living matter. Science, 1978, 200: 1012-1018

17 Rose G D, Geselowitz A R, Lesser G J, Lee R H, Zehfus M H. Hydrophobicity of amino acid residues in globular proteins. Science, 1985, 229: 834-838[DOI]

18 Ladiwala A, Xia F, Luo Q, Breneman C M, Cramer S M. Investigation of protein retention and selectivity in HIC systems using quantitative structure retention relationship models. Biotech Bioengin, 2006, 93(5): 836-850[DOI]

19 Maiorov V, Abagyan R. Energy strain in three-dimensional protein structures. Folding \& Design, 1998, 3: 259-269[DOI]

20 Eisenhaber F, Argos P. Improved strategy in analytic surface calculation for molecular systems: handling of singularities and computational efficiency. J Comput Chem, 1993, 14(11): 1272-1280[DOI] 